\title{
Optimal Design of Inverse Electromagnetic Problems with Uncertain Design Parameters Assisted by Reliability and Design Sensitivity Analysis
}

\author{
Ziyan Ren ${ }^{1,2}$, Doojong Um², and Chang-Seop Koh ${ }^{2 *}$ \\ ${ }^{1}$ School of Electrical Engineering, Shenyang University of Technology, Shenyang 110870, China \\ ${ }^{2}$ College of Electrical and Computer Engineering, Chungbuk National University, Chungbuk 361-763, Korea
}

(Received 6 November 2013, Received in final form 10 June 2014, Accepted 11 June 2014)

\begin{abstract}
In this paper, we suggest reliability as a metric to evaluate the robustness of a design for the optimal design of electromagnetic devices, with respect to constraints under the uncertainties in design variables. For fast numerical efficiency, we applied the sensitivity-assisted Monte Carlo simulation (S-MCS) method to perform reliability calculation. Furthermore, we incorporated the S-MCS with single-objective and multi-objective particle swarm optimization algorithms to achieve reliability-based optimal designs, undertaking probabilistic constraint and multi-objective optimization approaches, respectively. We validated the performance of the developed optimization algorithms through application to the optimal design of a superconducting magnetic energy storage system.
\end{abstract}

Keywords : inverse electromagnetic problem, Monte Carlo simulation, reliability evaluation, sensitivity analysis

\section{Introduction}

Recent optimal design algorithms of inverse electromagnetic problems have paid attention to uncertainties in design variables caused by, for example, manufacturing tolerance, uneven material properties, and the imperfect control of operating conditions. These uncertainties often force the deterministic optimal design to violate some constraints, by moving it to the infeasible region $[1,2]$. To deal with uncertainties, robust optimal design methods have been developed to improve product quality, by minimizing variations of the system performance [2-4]. However, a reliable algorithm that guarantees the constraint condition in probabilistic terms against uncertain design variables has not yet been popularly presented in the area of electrical engineering. In the fields of mechanical and structural design, the concept of making a trade-off between performance and reliability has recently been addressed, to increase the robustness of constraint functions [5-7].

In this paper, the reliability of a design is defined as the probability of remaining in the feasible region with respect to a constraint function, when the design is perturbed by uncertainties in the design variables. In mechanical engineering, there have been numerical attempts to evaluate reliability,

CThe Korean Magnetics Society. All rights reserved.

*Corresponding author: Tel: +82-43-274-2426

Fax:+82-43-274-2426, e-mail: kohcs@chungbuk.ac.kr such as Monte Carlo simulation (MCS), and first-order reliability methods (FORM) [7]. The MCS method is a sampling-based method, and it is known to be accurate only if the number of samples is large enough. Due to the huge computational effort, this method is not practical for engineering problems that normally involve performance analysis by numerical methods, such as the finite element method (FEM). The FORM is an optimization-based method, which calculates the reliability based on the shortest distance from a design to a constraint surface, in a normalized design space. This method, however, is also expensive to apply to an engineering problem, since the reliability calculation itself needs to solve another independent optimization problem.

It has been difficult to find a practical and guaranteed reliability calculation algorithm that can be applied to a reliability-based optimal design of electromagnetic devices subject to performance related constraints that involve numerical analysis methods, such as the FEM.

In this paper, in order to achieve a constraint-reliable optimal design against uncertain design variables, the sensitivity-assisted Monte Carlo simulation method is applied, for numerically efficient reliability calculation. Furthermore, based on the fast reliability calculation, constraint reliabilitybased and multi-objective reliability-based optimal design algorithms are developed. The validity of the developed algorithms is investigated through applications to an analytic example, and to a superconducting magnetic energy storage 


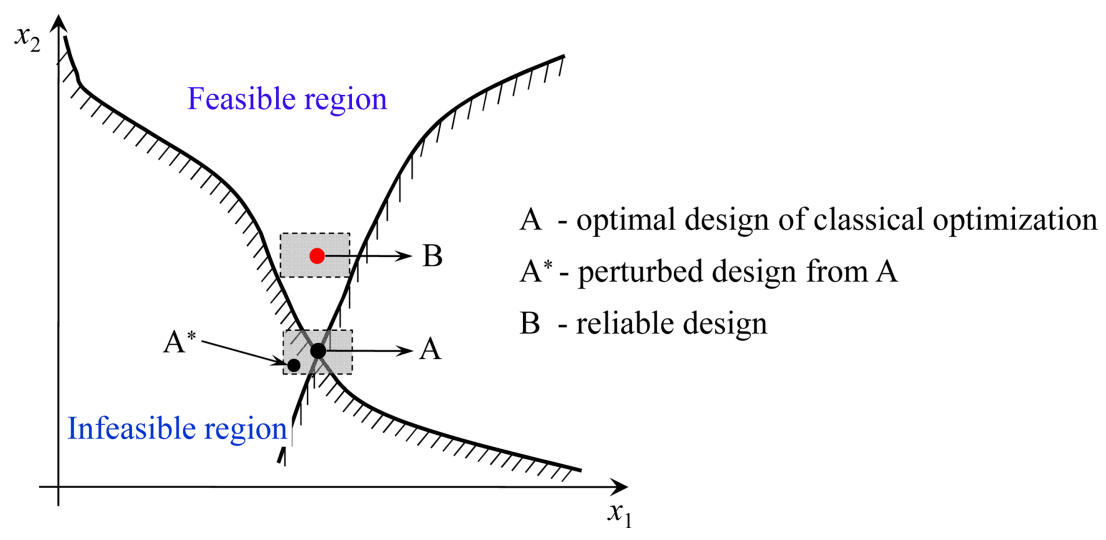

Fig. 1. (Color online) Optimal designs with uncertain design variables, where the filled gray regions are uncertain ranges.

(SMES) system.

\section{Classical/Deterministic Optimization Algorithm}

In a classical optimization problem that does not take into account any uncertainties in design variables, the mathematic optimization model is formulated as follows - for example, when the objective function $f(\cdot)$ is to be minimized, subject to a set of constraints $g(\cdot) \geq 0$ :

$$
\begin{array}{ll}
\text { Minimize } & f(\mathbf{d}) \\
\text { subject to } & g_{i}(\mathbf{d}) \geq 0, \quad i=1, \ldots, M \\
& \mathbf{d}_{L} \leq \mathbf{d} \leq \mathbf{d}_{U}, \mathbf{d} \in R^{N}
\end{array}
$$

where, $\mathbf{d}=\left[d_{1}, d_{2}, \ldots, d_{N}\right]^{T}$ is the vector of deterministic design variables of which every element does not include uncertainty; $\mathbf{d}_{L}$ and $\mathbf{d}_{U}$ are the upper and lower bounds, respectively, and $M$ is the number of constraint functions.

This method often locates its optimal design near to, or on the constraint surface, as shown in Fig. 1. If the design is perturbed by a small variation, the deterministic optimum may easily move to the infeasible region, such as design $\mathrm{A}^{*}$ in Fig. 1, to violate one or more constraints. On the other hand, design B in Fig. 1 is considered a reliable optimal design, because it will remain in the feasible region, even with uncertainties in the design variables. In the robust optimization and reliability-based optimizations, the reliable design $\mathrm{B}$ is preferred to design $\mathrm{A}$, even though its performance is not as satisfactory as that of $\mathrm{A}$.

\section{Reliability Based Optimal Design Algorithms}

For a specified engineering problem, the modeling of uncertainty needs a lot of information. In this paper, for simplicity, the following assumptions are made:

1) For reliability analysis, every design variable has un- certainty that follows a Gaussian distribution with zero mean value and standard deviation $(\sigma)$, and the design is described as $\mathbf{x}$. Based on the confidence interval, for a given design $\mathbf{x}=\left[x_{1}, x_{2}, \ldots, x_{N}\right]^{T}$, the uncertainty set (or uncertain range) $U(\mathbf{x})$ is defined as follows:

$$
U(\mathbf{x})=\left\{\xi \in R^{N} \mid \mathbf{x}-k \boldsymbol{\sigma} \leq \xi \leq \mathbf{x}+k \boldsymbol{\sigma}\right\}
$$

where, $\xi$ is any perturbed design, and $k$ is a constant that is decided according to the required confidence level. For example, $k$ will be 1.96 , for the confidence level of $95 \%$.

2) The uncertainties are independent of each other.

Based on the above assumptions, two reliability-based optimal design (RBOD) algorithms are investigated below.

\subsection{Probabilistic Constraint Approach (PCA)}

In this method, the reliability of a design is treated as probabilistic constraints, so that the reliability for each constraint may be higher than or equal to a required value. The formulation of the deterministic optimization problem (1) is changed as follows:

$$
\begin{array}{ll}
\text { Minimize } & f(\mathbf{x}) \\
\text { subject to } & R\left(g_{i}(\mathbf{x}) \geq 0\right) \geq R_{t, i}, \quad i=1, \ldots, M \\
& \mathbf{x}_{L} \leq \mathbf{x} \leq \mathbf{x}_{U}, \mathbf{x} \in R^{N}
\end{array}
$$

where, $R_{t, i}$ is the target reliability for the $i$-th constraint function, which will be decided by the confidence level of the optimal design.

By solving (3) using a global optimization method, such as particle swarm optimization (PSO) and genetic algorithms, a global constraint-reliable optimal design will be obtained, which guarantees the target reliability for each constraint.

\subsection{Multi-Objective Functions Approach (MOFA)}

This method treats the reliability as another independent objective function to be maximized. For a problem including multiple constraint functions, the system reliability is 
defined as the minimum one among all the constraint reliabilities.

In considering optimizing the targets of nominal objective function and system reliability, the deterministic optimization problem (1) is transferred into:

$$
\begin{array}{ll}
\text { Minimize } & f(\mathbf{x}) \\
\text { Maximize } & R_{\min }=\min _{1 \leq i \leq M} R\left(g_{i}(\mathbf{x}) \geq 0\right) \\
\text { subject to } & \mathbf{x}_{L} \leq \mathbf{x} \leq \mathbf{x}_{U}, \mathbf{x} \in R^{N}
\end{array}
$$

In solution of (4), a multi-objective global optimization algorithm, such as the multi-guider cross-searching multiobjective PSO (MGC-PSO) algorithm [8] or non-dominated sorting genetic algorithm-II [9], can be applied.

It should be noted that this method gives a Pareto-optimal set, instead of just one global optimal solution, as in the PCA. The Pareto front includes designs, which range from the design with the best performance and lowest reliability, to that with the worst objective performance and highest reliability value. Therefore, a proper optimal design will be selected, according to a designer's requirement.

\section{Reliability Calculation Methods}

\subsection{Conventional Monte Carlo Simulation (MCS) Method}

In the conventional MCS method, the reliability of a given design $\mathbf{x}$ with respect to the $i$-th constraint $g_{i}(\mathbf{x}) \geq 0$ is numerically calculated, by using the following procedure.

1) Generate $N_{t}$ testing points in the uncertainty set $U(\mathbf{x})$, as shown in Fig. 2;

2) For all testing points, calculate the constraint function values, and check if they satisfy the $i$-th constraint function or not;

3) Evaluate the reliability, as follows:

$$
R\left(g_{i}(\mathbf{x}) \geq 0\right)=\frac{n}{N_{t}}
$$

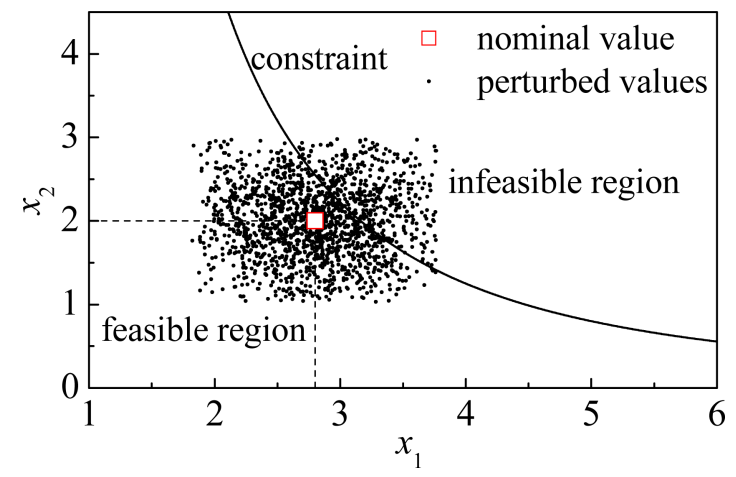

Fig. 2. (Color online) Monte Carlo simulation method, where 100,000 testing points are generated in the uncertain range, with the confidence level of $95 \%$, and $\sigma_{1}=\sigma_{2}=0.5$. where, $n$ is the number of testing points that satisfy the $i$ th constraint.

It is obvious from (5) that the accuracy of reliability prediction is proportional to the total number of testing points. Usually, as many testing points as possible are recommended; but this is almost impossible for an engineering application. The selection of $N_{t}$ is normally based on the probability of failure $\mathrm{pf}=1-R_{t}$. $N_{t}$ is generally equal to $100 /$ pf. For example, if the target reliability is $99 \%$, the number of test points should be at least 10,000. Therefore, if the required reliability is very high, it makes the method very time-consuming.

\subsection{Sensitivity-Assisted Monte Carlo Simulation (S- MCS) Method}

Thanks to modern mass production technologies, the standard deviation of the uncertain design variable will be kept very small. The corresponding uncertainty range is also quite narrow, even when the confidence level is very high (e.g., 99\%).

For a testing point $\xi \in U(\mathbf{x})$, the $i$-th constraint function value can be linearly approximated as follows:

$$
\begin{aligned}
& g_{i}(\boldsymbol{\xi}) \cong g_{i}(\mathbf{x})+\nabla g_{i}(\mathbf{x}) \cdot(\boldsymbol{\xi}-\mathbf{x}) \\
& \nabla g_{i}(\mathbf{x})=\left\{\frac{\partial g_{i}}{\partial x_{1}}, \frac{\partial g_{i}}{\partial x_{2}}, \cdots, \frac{\partial g_{i}}{\partial x_{N}}\right\}^{T}
\end{aligned}
$$

where, the gradient vector of the constraint function $g_{i}(\mathbf{x})$ is calculated by using design sensitivity analysis with the help of the FEM, as follows [10]:

$$
\begin{aligned}
& \nabla g_{i}(\mathbf{x})=\left.\frac{\partial g_{i}}{\partial \mathbf{x}^{T}}\right|_{\mathbf{A}=C}-[\lambda]^{T}\left(\left.\frac{\partial \mathbf{R}}{\partial \mathbf{x}^{T}}\right|_{\nu=C}-\frac{\partial \mathbf{R}}{\partial v} \frac{\partial v}{\partial B^{2}} \frac{\partial B^{2}}{\partial \mathbf{x}^{T}}\right) \\
& {[\mathbf{K}+\overline{\mathbf{K}}]^{T}[\lambda]=\frac{\partial g_{i}}{\partial \mathbf{A}^{T}}}
\end{aligned}
$$

where, $\mathbf{R}$ is the residual vector from the Galerkin approximation; $v$ is the non-linear magnetic reluctivity; $\mathbf{A}$ is the magnetic vector potential; $\lambda$ is the adjoint variable; $\mathbf{K}$ and $\overline{\mathbf{K}}$ are linear and non-linear parts of the stiffness matrix; and other symbols have their usual meanings in the FEM. Once the gradient vector of the constraint function is computed from (7) and (8), the constraint function can be treated as an analytic one, so that using (5), the reliability evaluation will be efficiently performed.

The evaluation of the reliability in this method consumes at most around $(1+M)$ times of analyses, using the FEM without regard to the number of testing points; while the conventional MCS requires as many as the number of testing points. This reveals that the proposed S-MCS method is numerically efficient. For some problems in which design sensitivity analysis by FEM is not available, or for largescale nonlinear problems, the Kriging surrogate model can 


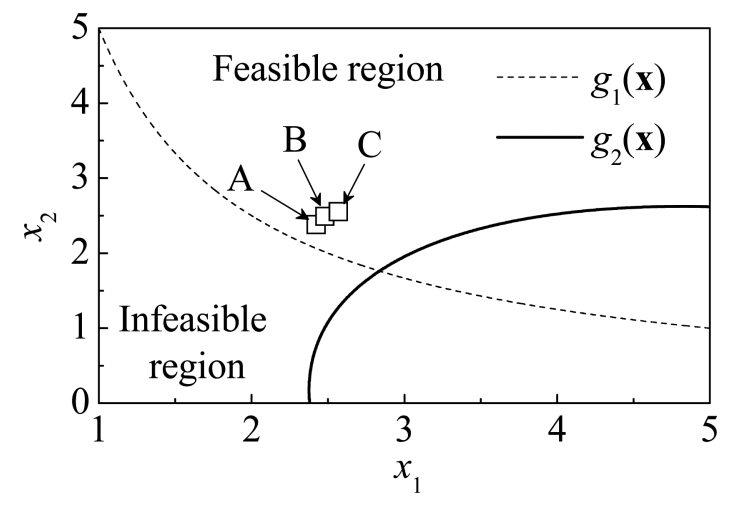

Fig. 3. Constraint functions.

be used to perform performance and sensitivity approximation [11], so as to decrease expensive cost for reliability analysis and optimization.

\subsection{Reliability Calculation Results}

In order to check the accuracy of the reliabilities calculated from the proposed S-MCS method, two geometric constraints, as shown in Fig. 3, with two uncertain design variables, $x_{i} \sim N\left(\mu_{i}, \sigma_{i}=0.3\right),(i=1,2)$, are investigated as:

$$
\begin{aligned}
& g_{1}(\mathbf{x})=\frac{x_{1} x_{2}}{5}-1 \geq 0 \\
& g_{2}(\mathbf{x})=\frac{\left(x_{1}+x_{2}-5\right)^{2}}{30}+\frac{\left(x_{1}-x_{2}-12\right)^{2}}{120}-1 \geq 0
\end{aligned}
$$

The gradient vectors of the two constraints are derived as follows:

$$
\begin{aligned}
& \nabla g_{1}(\mathbf{x})=\left\{\frac{x_{2}}{5}, \frac{x_{1}}{5}\right\}^{T} \\
& \nabla g_{2}(\mathbf{x})=\left\{\frac{5 x_{1}+3 x_{2}-32}{60}, \frac{3 x_{1}+5 x_{2}-8}{60}\right\}^{T}
\end{aligned}
$$

In the reliability calculation, the confidence level and the number of testing points are set to $95 \%$ and one million,

Table 1. Comparison of reliability calculations.

\begin{tabular}{ccccc}
\hline \hline \multirow{2}{*}{ Constraints } & \multirow{2}{*}{ Designs* } & \multicolumn{3}{c}{ Reliability $(R)$} \\
\cline { 3 - 5 } & & MCS & S-MCS & Error (\%) \\
\hline \multirow{3}{*}{$g_{1}(\mathbf{x}) \geq 0$} & A & 0.7967 & 0.7992 & 0.314 \\
& B & 0.9006 & 0.8946 & 0.666 \\
& C & 0.9585 & 0.9500 & 0.886 \\
\hline \multirow{3}{*}{$g_{2}(\mathbf{x}) \geq 0$} & A & 0.9989 & 0.9979 & 0.100 \\
& B & 0.9995 & 0.9988 & 0.070 \\
& C & 0.9992 & 0.9984 & 0.080 \\
\hline
\end{tabular}

Function calls $\quad 1,000,000 \quad 2$ -

*A(2.423,2.382), $\mathrm{B}(2.480,2.490)$ and $\mathrm{C}(2.568,2.554)$. respectively.

For the three test designs $\mathrm{A}, \mathrm{B}$, and $\mathrm{C}$ in Fig. 3, the reliabilities are calculated by using the MCS and the proposed S-MCS methods, and are compared in Table 1. It is found that the reliabilities calculated from S-MCS show good agreement with those from MCS, with the maximum error of $0.886 \%$.

\section{Optimization Results of SMES}

The developed reliability-based global optimization algorithms are applied to TEAM problem 22, the optimal design of a superconducting magnetic energy storage system shown in Fig. 4 [12]. The optimization target is to find the best combination of the geometric parameters $\left(R_{1}\right.$, $\left.H_{1}, D_{1}, R_{2}, H_{2}, D_{2}\right)$ and current densities $\left(J_{1}, J_{2}\right)$ of current carrying coils, by satisfying the following requirements:

- The stored total energy is $E_{r e f}=180 \mathrm{MJ}$;

- The magnetic stray field $B_{\text {stray }}$ evaluated by 21 sampling points on lines a and $\mathrm{b}$ marked in Fig. 4, is minimized.

Furthermore, the problem should satisfy the following constraints, as shown in Fig. 5, to maintain the superconducting state of the coils:

$$
g_{i}(\mathbf{x})=54-\left|J_{i}\right|-6.4\left|B_{\max , i}(\mathbf{x})\right| \geq 0, \quad i=1,2
$$

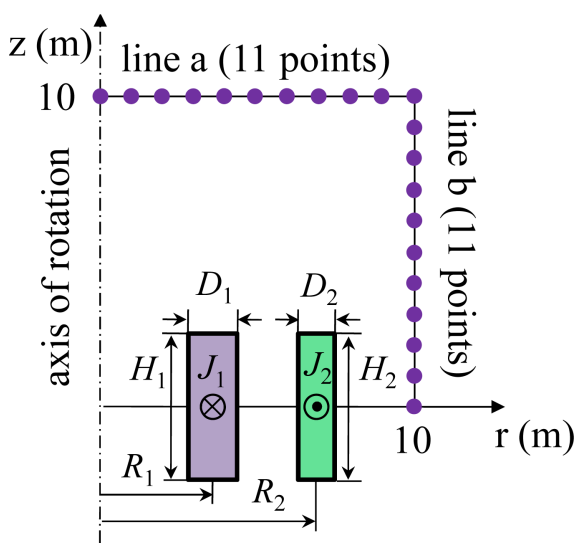

Fig. 4. (Color online) Configuration of SMES system.

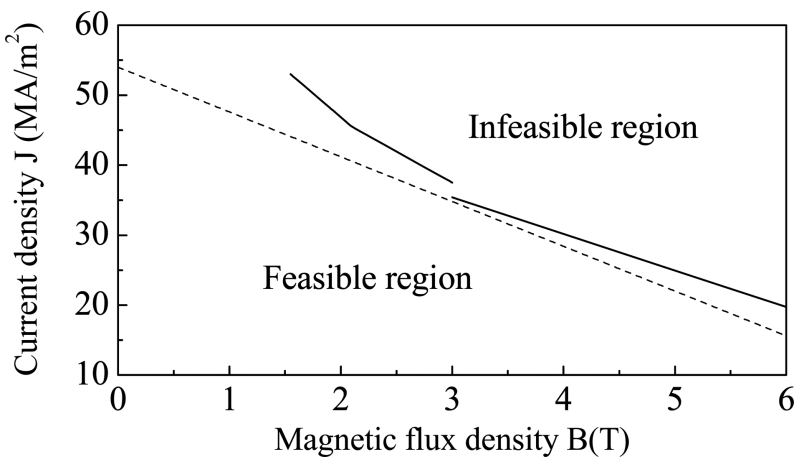

Fig. 5. Quenching curve of NbTi-superconductor. 
where, $B_{\max , i}$ is the maximum magnetic flux density in the $i$-th coil.

In the classical/deterministic optimization, the optimization problem is formulated as:

$$
\begin{aligned}
\text { Minimize } \quad f(\mathbf{x}) & =\frac{B_{\text {stray }}^{2}}{B_{\text {norm }}^{2}}+\frac{\left|E(\mathbf{x})-E_{\text {ref }}\right|}{E_{\text {ref }}} \\
\text { subject to } \quad g_{i}(\mathbf{x}) & =54-\left|J_{i}\right|-6.4\left|B_{\text {max }, i}(\mathbf{x})\right| \geq 0, \quad i=1,2 \\
g_{3}(\mathbf{x}) & =\left(R_{2}-\frac{D_{2}}{2}\right)-\left(R_{1}+\frac{D_{1}}{2}\right) \geq 0 \\
B_{\text {stray }}^{2} & =\sum_{i=1}^{21} B_{s, i}^{2}(\mathbf{x})
\end{aligned}
$$

where, $B_{s, i}$ is the magnetic stray field at the $i$-th sampling point, and the reference stray field is $B_{\text {norm }}=1 \mathrm{mT}$. The third constraint is to keep the two coils from overlapping.

The current densities are usually controlled by a current controller. When it needs to compensate a perturbation, current densities are limited within a certain range. Therefore, the current densities may deviate from the nominal values with an unavoidable range. In this paper, uncertainties in the current densities $\mathbf{J}=\left(J_{1}, J_{2}\right)^{T}$ are considered in the optimal design, with the assumption that they follow Gaussian distributions with nominal values of $\mathbf{J}_{0}=(16.78$, $-15.51)^{T} \mathrm{MA} / \mathrm{m}^{2}$, and standard deviations of $\boldsymbol{\sigma}=(0.179$, $0.179)^{T} \mathrm{MA} / \mathrm{m}^{2}$, respectively. The geometric variables $\left(R_{2}\right.$, $\mathrm{H}_{2}$, and $\mathrm{D}_{2}$ ) are taken as deterministic design variables, with their design ranges of $1.8 \mathrm{~m} \leq R_{2} \leq 5.0 \mathrm{~m}, 0.2 \mathrm{~m} \leq H_{2}$ $\leq 3.6 \mathrm{~m}$, and $0.1 \mathrm{~m} \leq D_{2} \leq 0.8 \mathrm{~m}$, respectively. Other geometric design variables are fixed to $R_{1}=1.32 \mathrm{~m}, H_{1}=$ $2.14 \mathrm{~m}$, and $D_{1}=0.59 \mathrm{~m} \mathrm{[1]}$.

The accuracy and efficiency of the S-MCS method have been discussed and compared with the conventional MCS method and the FORM method in detail in Ref. [13], which also investigated both the three- and eight- parameter SMES problems. Therefore, this section only focuses on comparison with different formulations of reliability-based optimization.

In reliability-based optimization, the optimal design problem is formulated with the design variable vector of $\mathbf{x}=$ $\left(R_{2}, H_{2}, D_{2}, J_{1}, J_{2}\right)^{\mathrm{T}}$, as follows:

- PCA-RBOD algorithm

Minimize $f(\mathbf{x})=\frac{B_{\text {stray }}^{2}}{B_{\text {norm }}^{2}}+\frac{\left|E(\mathbf{x})-E_{\text {ref }}\right|}{E_{\text {ref }}}$

subject to $R\left(g_{i}(\mathbf{x})=54-\left|J_{i}\right|-6.4\left|B_{\max , i}(\mathbf{x})\right| \geq 0\right) \geq R_{t, i}, i=1,2(13)$

$$
g_{3}(\mathbf{x})=\left(R_{2}-\frac{D_{2}}{2}\right)-\left(R_{1}+\frac{D_{1}}{2}\right) \geq 0
$$

where, $R_{t, i}$ is the target reliability of the $i$-th constraint function.

- MOFA-RBOD algorithm

$$
\begin{aligned}
\text { Minimize } f(\mathbf{x}) & =\frac{B_{\text {stray }}^{2}}{B_{\text {norm }}^{2}}+\frac{\left|E(\mathbf{x})-E_{\text {ref }}\right|}{E_{\text {ref }}} \\
\text { Maximize } R_{\min } & =\min _{1 \leq i \leq 2} R\left(g_{i}(\mathbf{x})=54-\left|J_{i}\right|-6.4\left|B_{\text {max }, i}(\mathbf{x})\right| \geq 0\right) \\
\text { subject to } g_{i}(\mathbf{x}) & =54-\left|J_{i}\right|-6.4\left|B_{\text {max }, i}(\mathbf{x})\right| \geq 0, \quad i=1,2 \\
g_{3}(\mathbf{x}) & =\left(R_{2}-\frac{D_{2}}{2}\right)-\left(R_{1}+\frac{D_{1}}{2}\right) \geq 0
\end{aligned}
$$

For the reliability calculation using the suggested S-MCS method, the constraints are approximated as follows:

$$
g_{i}(\boldsymbol{\xi}) \cong g_{i}(\mathbf{x})+\left.\frac{\partial g_{i}}{\partial \mathbf{J}^{\mathrm{T}}}\right|_{\mathbf{J}=\mathbf{J}_{0}} \cdot\left(\mathbf{J}-\mathbf{J}_{0}\right), \quad i=1,2
$$

where, the gradient vector of the constraint function with respect to current density is obtained by using sensitivity analysis and the FEM, as follows:

$$
\begin{aligned}
& {[\mathbf{K}][\mathbf{A}]=\{\mathbf{Q}\}} \\
& \frac{d B_{\text {max }, i}}{d \mathbf{J}^{T}}=\left.\frac{\partial B_{\text {max }, i}}{\partial \mathbf{J}^{T}}\right|_{A=\text { constant }}+\left[\boldsymbol{\lambda}_{i}\right]^{T} \frac{\partial\{\mathbf{Q}\}}{\partial \mathbf{J}^{T}}, i=1,2 \\
& {[\mathbf{K}]^{T}\left[\boldsymbol{\lambda}_{i}\right]=\frac{\partial B_{\text {max }, i}}{\partial \mathbf{A}^{T}}, \quad i=1,2 .}
\end{aligned}
$$

where, Eq. (16-a) is the system matrix, $\mathbf{Q}$ is the forcing vector, and the other symbols have the same meanings as explained in (7) and (8).

The reliabilities are calculated by using the proposed SMCS method with the confidence level and number of testing points of $95 \%$ and one million, respectively. The PSO with initial 30 particles and 200 maximum iterations is applied for the solution of Eqs. (12) and (13), while the MGC-PSO with initial 50 particles and 300 maximum iterations is applied for Eq. (14) [8].

Table 2 compares the optimal designs from the proposed PCA-RBOD algorithm under different target reliabilities, with that obtained from the classical global optimization. It is found that the classical optimal design has very low reliability, and it has higher possibility, in this case $44 \%$, of violating the constraint for keeping the superconducting state. In general, as the target reliability increases, the PCARBOD tends to locate its optimum further inside the feasible region.

Figs. 6(a) and (b) show the Pareto-optimal designs obtained from the proposed MOFA-RBOD method, together with the optimal solutions in Table 2. From Fig. 6(b), it is found that the Pareto-front includes the optimal solutions obtained from the PCA-RBOD with different target reliabilities, and the design A (one of the extreme solutions) is very similar to the classical optimal design. The Pareto-front also provides important information for making a balance between objec- 
Table 2. Comparison of the reliability-based optimal design results.

\begin{tabular}{|c|c|c|c|c|c|c|c|c|c|c|}
\hline \multirow{2}{*}{$R_{t}$} & \multicolumn{3}{|c|}{ Optimal design [m] } & \multirow{2}{*}{$\begin{array}{c}E \\
{[\mathrm{MJ}]}\end{array}$} & \multirow{2}{*}{$\begin{array}{l}B_{\text {stray }} \\
{[\mu \mathrm{T}]}\end{array}$} & \multirow{2}{*}{$f$} & \multicolumn{2}{|c|}{ Constraints } & \multirow{2}{*}{$R\left(g_{1} \geq 0\right)$} & \multirow{2}{*}{ FEM calls } \\
\hline & $R_{2}$ & $\mathrm{H}_{2} / 2$ & $D_{2}$ & & & & $g_{1}$ & $g_{2}$ & & \\
\hline Classical & 1.8127 & 1.4963 & 0.2458 & 180.00 & 40.6813 & $1.71 \mathrm{E}-3$ & 0.1191 & 3.0801 & 0.5604 & 3600 \\
\hline 0.65 & 1.8106 & 1.4718 & 0.2506 & 178.36 & 68.7756 & $1.39 \mathrm{E}-2$ & 0.3864 & 2.6574 & 0.6884 & 4770 \\
\hline 0.75 & 1.8068 & 1.4647 & 0.2528 & 176.84 & 86.0335 & $2.36 \mathrm{E}-2$ & 0.5550 & 2.4047 & 0.7609 & 4860 \\
\hline 0.90 & 1.8005 & 1.4869 & 0.2514 & 175.52 & 187.859 & $6.02 \mathrm{E}-2$ & 0.5615 & 2.3596 & 0.9361 & 5040 \\
\hline 0.95 & 1.8000 & 1.5274 & 0.2488 & 176.44 & 1280.79 & $1.66 \mathrm{E}+0$ & 0.6430 & 2.4622 & 0.9614 & 5400 \\
\hline
\end{tabular}

*The reliability for the constraint $g_{2}(\mathbf{x}) \geq 0$ is 1.0 for all cases.

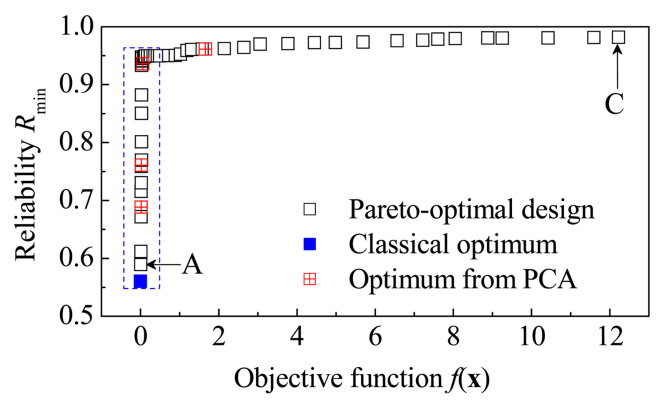

(a) Pareto-optimal designs

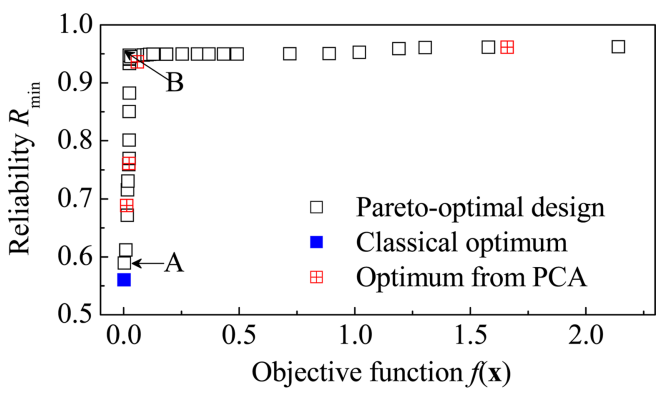

(b) Pareto-front in the reduced space

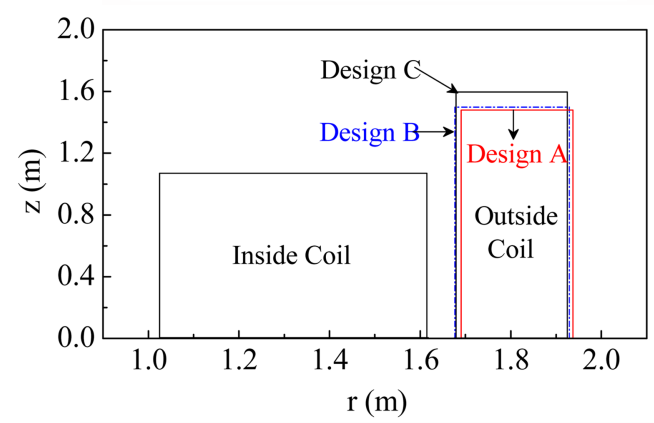

(c) Optimal configurations of coils

Fig. 6. (Color online) Comparison of the optimal results.

tive function and reliability, according to different requirements. For example, when the constraints are extremely critical, design $\mathrm{C}$ may be selected, although it has very poor objective value. Design B $\left(f=2.55 \mathrm{E}-2, R_{\min }=0.9475\right)$ may be considered as a good solution in general purpose optimization, since it makes a good trade-off between performance and reliability. Fig. 6(c) compares the configurations of the coils for three representative optimal designs A, B, and C.

\section{Conclusions}

In this paper, with the presence of uncertainties in design variables, two reliability-based optimal design (RBOD) algorithms, those of probabilistic constraint (PCA) and multiobjective functions approaches (MOFA) are developed, based on the sensitivity-assisted reliability calculation method (SMCS), and applied to the optimal design of an electromagnetic device. From comparisons of optimization results, the following conclusions are made:

The proposed reliability calculation method, S-MCS, speeds up the reliability calculation, especially for the performance related constraints, with high accuracy, under relatively small standard deviation. In addition, the PCA-RBOD provides only one reliable optimal design for specific target reliability; while the Pareto-front from MOFA-RBOD gives much more assistance to a designer, in establishing a trade-off between performance and reliability.

\section{Acknowledgement}

This research was supported by the Basic Science Research Program, through the NRF of Korea, funded by the Ministry of Education, Science and Technology (2011-0013845).

\section{References}

[1] G. Steiner, A. Weber, and C. Magele, IEEE Trans. Magn. 40, 1094 (2004).

[2] Z. Y. Ren, M. T. Pham, M. H. Song, D. H. Kim, and C. S. Koh, IEEE Trans. Magn. 47, 1254 (2011).

[3] F. G. Guimaraes, D. A. Lowther, and J. A. Ramirez, IEEE Trans. Magn. 42, 1207 (2006).

[4] L. Egiziano, P. Lamberti, G. Sagnuolo, and V. Tucci, IEEE Trans. Magn. 44, 1134 (2008).

[5] X. P. Du, A. Sudjianto, and W. Chen, ASME J. Mech. Des. 126, 562 (2004).

[6] W. Chen, M. M. Wiecek, and J. Zhang, ASME J. Mech. Des. 121, 179 (1999). 
[7] K. Deb, IEEE Trans. Evol. Comput. 13, 1054 (2009).

[8] M.-T. Pham, D. H. Zhang, and C.-S. Koh, IEEE Trans. Magn. 48, 539 (2012).

[9] K. Deb, A. Pratap, S. Agrawal, and T. Meyarivan, IEEE Trans. Evol. Comput. 6, 182 (2002).

[10] J. S. Ryu, Y. Yao, C. S. Koh, S. Yoon, and D. S. Kim, IEEE Trans. Magn. 41, 1792 (2005).
[11] B. Xia, Z. Y. Ren, and C.-S. Koh, IEEE Trans. Magn. 50, 7017104 (2014)

[12] http://www.igte.tugraz.at/archive/team_new/ description.php.

[13] Z. Y. Ren, D. H. Zhang, and C.-S. Koh, J. Electr. Eng. Technol. 8, 331 (2013). 